Revue des patrimoines

$21 \mid 2013$

De l'art de bâtir aux champs à la ferme moderne

\title{
Les constructions agricoles contemporaines : des objets discrets de la modernisation agricole
}

\section{Philippe Madeline}

\section{OpenEdition}

\section{Journals}

Édition électronique

URL : http://journals.openedition.org/insitu/10360

DOI : $10.4000 /$ insitu. 10360

ISSN : 1630-7305

\section{Éditeur}

Ministère de la culture

Référence électronique

Philippe Madeline, «Les constructions agricoles contemporaines : des objets discrets de la modernisation agricole », In Situ [En ligne], 21 | 2013, mis en ligne le 10 juillet 2013, consulté le 19 avril 2019. URL : http://journals.openedition.org/insitu/10360 ; DOI : 10.4000/insitu.10360

Ce document a été généré automatiquement le 19 avril 2019

\section{(c) $($ i) $(9)$}

In Situ Revues des patrimoines est mis à disposition selon les termes de la licence Creative Commons Attribution - Pas d'Utilisation Commerciale - Pas de Modification 4.0 International. 


\title{
Les constructions agricoles contemporaines : des objets discrets de la modernisation agricole
}

\author{
Philippe Madeline
}

\section{Introduction}

1 Dans leur analyse des mutations des campagnes métropolitaines après 1945, les géographes ruralistes ont privilégié l'élan de modernisation agricole impulsé par les pouvoirs publics. La diminution du nombre d'exploitations, la spécialisation des productions, la mécanisation et la motorisation des activités, les avancées en matière de productivité ou encore l'amélioration des conditions de travail sont des thèmes récurrents des thèses d'État soutenues au lendemain de la Seconde Guerre mondiale jusqu'au début des années 1990. Certains aspects, plus que d'autres, ont retenu l'attention des chercheurs. La motorisation des tâches incarnée par l'essor du tracteur en est un. Au regard des éléments du système d'exploitation, les bâtiments agricoles ont joué un rôle clé que les géographes n'ont pas omis de mentionner. Pour autant, rares sont ceux qui leur ont accordé une place de choix dans l'analyse de la modernisation.

Pourquoi la construction agricole reste-t-elle un aspect relativement oublié ? Au-delà de l'inégale ampleur des constructions liée à l'intensité des transformations et des spécialisations, plusieurs éléments d'explication peuvent être avancés. En effet, si les géographes n'ont pas manqué de souligner l'inadaptation des constructions anciennes aux exigences de l'agriculture moderne, les changements propres à l'évolution de leur discipline ont eu aussi une incidence. Après des décennies où l'analyse de la maison paysanne fut emblématique de la géographie française, l'ampleur des changements survenus dans les campagnes a pu réduire l'intérêt géographique de l'objet. Enfin pour les nouvelles générations de chercheurs attirés par de nouvelles méthodes et échelles 
d'analyse, la faible disponibilité des sources statistiques a pu réduire l'intérêt de leur analyse.

\section{Quelle place pour les constructions dans l'analyse de la modernisation agricole?}

3 Parmi les géographes ruralistes des années d'après-guerre au début des années 1990, rares sont ceux qui ne se sont pas intéressés aux activités agricoles. Dans les thèses sur les ressorts de la modernisation agricole ou dans les thèses régionales, les géographes ont scruté les mutations agricoles sous toutes leurs formes. Parmi la trentaine de doctorats d'État soutenus entre 1946 et 1992 sur lesquels s'appuie cet article, les géographes ont analysé différentes facettes de la modernisation en privilégiant certains thèmes: la diminution des structures d'exploitation et leur agrandissement, les restructurations foncières, la spécialisation des productions, la motorisation ou encore l'utilisation des intrants chimiques. Les traces relatives à la construction de nouveaux bâtiments sont beaucoup plus ténues même si les géographes s'accordent tous pour en distinguer l'importance. Comment pourrait-il en être autrement? Les constructions agricoles font partie intégrante du capital d'exploitation.

\section{Les bâtiments agricoles : une clé de l'intégration des exploitations au modèle productiviste}

4 Les bâtiments sont constitutifs des structures agraires, entendues comme «l'ensemble des conditions foncières et sociales d'un espace agricole ${ }^{1} »$. À ce titre, ils forment une partie du capital d'une exploitation agricole que l'on divise traditionnellement en trois éléments : le capital foncier (la terre); le capital d'exploitation composé du matériel agricole (le cheptel mort), du bétail (le cheptel vif) et des bâtiments agricoles et le capital financier. À proximité ou à distance, des bâtiments accompagnent donc, sauf exception, chaque domaine en activité. Ils traduisent les orientations de production et l'importance économique de l'exploitation. Selon l'orientation considérée, leur rôle présente des réalités multiples: un local de stockage des produits, un abri pour les animaux et le matériel agricole, un lieu consacré à l'élevage et à la transformation de la production. C'est dans le cadre de l'élevage que leur importance est la plus forte : ils sont l'une des composantes «qui contribuent le plus à l'endettement mais également [celles qui] influent le plus sur les gains de productivité ${ }^{2}$ ».

5 Chaque bâtiment apparait comme un élément du "système de production » qui caractérise une combinaison de productions et de moyens mis en œuvre pour les obtenir. Une exploitation s'appuie alors sur trois types de ressources : des ressources foncières ou hydrauliques, de la main-d'œuvre et le capital d'exploitation. Parmi ces moyens de production, le bâti des exploitations tient un rôle clé pour les structures d'élevage. Il est un élément du « système d'élevage » qu'Armand Frémont définit comme « la combinaison de techniques agricoles consacrées à l'élevage, des produits qui en résultent et des modes de commercialisation de ces produits, le système d'élevage permet de définir l'activité principale d'une exploitation, d'une association d'exploitations et d'une région où l'élevage prédomine ${ }^{3} »$. 
6 Dans une approche à grande et moyenne échelles, l'auteur souligne l'interdépendance entre deux groupes d'éléments : d'une part, les techniques de production dans lesquelles figurent les modes de stabulation (et l'utilisation du sol, l'alimentation des animaux, les techniques de reproduction et de sélection des animaux) et, par ailleurs, les produits, les techniques de gestion, ainsi que les modes de commercialisation. Un système d'élevage recouvre donc à la fois des données économiques, agronomiques et techniques.

7 Une décennie plus tard et à une toute autre échelle, le géo-agronome Jean-Pierre Deffontaines décrypte, à partir de travaux menés dans des exploitations d'élevage des Vosges, l'incidence des innovations techniques sur l'agriculture ${ }^{4}$. Il mentionne que les bâtiments figurent en bonne place à l'intérieur du "système famille-exploitation ", qu'ils sont directement liés aux orientations productives - en l'occurrence ici le cheptel de vaches laitières - et s'inscrivent dans la stratégie familiale d'évolution de l'exploitation. Dans une approche complémentaire, l'auteur dégage à moyenne échelle les interactions de l'exploitation et de son environnement dans lesquelles le bâti est à la fois le produit de décisions internes à l'exploitation mais dépend aussi, dans le même temps, d'éléments extérieurs: le marché foncier, la capacité d'investissement, etc. Enfin, le système présenté est producteur « d'effets sur l'environnement »: donnée d'importance pour les géographes à travers le rôle des constructions agricoles dans la production de paysage. Dans une publication ultérieure, le même auteur insiste sur le fait que « l'espace bâti fait partie intégrante de l'espace d'activité » et déclare: «Nous aborderons peu les espaces bâtis mais ils devraient plus souvent être pris en compte simultanément à l'espace foncier car une modification de l'activité dans l'un entraîne une évolution dans l'autre ${ }^{5}$ ».

De nombreux travaux ont donc montré l'importance des bâtiments dans la production agricole dans la mesure où ils s'inscrivent comme l'un des facteurs de l'intégration des structures de production dans le modèle productiviste. Pour autant, cette cible a rarement été privilégiée par les auteurs. Dans le corpus étudié, les travaux qui ont investi ce vecteur de modernisation sont difficiles à trouver. Il faut attendre la fin des années 1960 et surtout les années 1970 pour que les auteurs accordent quelques lignes, voire un chapitre, à ce thème. Pour autant, les bâtiments agricoles modernes ne sont pas absents du propos : ils apparaissent nettement en creux, quand chaque auteur identifie le bâti agricole ancien comme un objet obsolète, comme un obstacle à la modernisation agricole.

\section{Les géographes et la construction agricole contemporaine : une approche par défaut}

9 Dans la France d'après-guerre, l'inadéquation des structures bâties héritées du passé éclate face aux nécessités de la modernisation et des choix économiques. À l'échelle nationale, $75 \%$ des bâtiments agricoles datent d'avant 1939 et plus de $42 \%$ des installations agricoles sont sorties de terre avant 1871. Le pourcentage s'élève davantage encore en Normandie, en Poitou-Charentes et dans l'Est. Dans le même temps, les bâtiments agricoles de moins de 25 ans ne représentent que le quart du total, à l'exception de la Côte-d'Azur et de la Bretagne dont le parc avait déjà été rajeuni ${ }^{6}$. Partout, les éleveurs se heurtent aux mêmes difficultés.

10 Pour le Morvan, Jacqueline Bonnamour signale en 1965 la vétusté de l'atelier agricole et l'insalubrité des étables, même si « l'arrivée de l'eau a été une façon de réduire la corvée de sortir les bêtes deux fois par jour pour les faire boire en hiver $»^{7}$. Dans les campagnes mancelles, Jeanne Dufour évoque quinze ans plus tard « l'exiguïté des bâtiments qui est la 
règle dans les pays de petites exploitations ». La difficulté pour l'exploitant, ajoute-t-elle, "est qu'il n'existe pas, le plus souvent, de bâtiments transformables " ${ }^{8}$. Dans le même temps, Georges Macé pour la Mayenne, relève d'insupportables conditions de travail : « II n'est pas possible de conduire 40 bonnes laitières ou d'engraisser chaque année une centaine de taurillons dans de vieux bâtiments inadaptés. Déjà un troupeau de 25 vaches laitières pour lequel on ne dispose que de l'étable archaïque, de l'ancienne écurie et peut-être d'un autre bâtiment de fortune, peut imposer d'insupportables conditions de vie à un couple d'agriculteurs. La vieille étable c'est une corvée ${ }^{9}$ !».

11 De la Basse-Normandie où Armand Frémont constate que « les étables ont été conçues pour des techniques totalement dépassées ${ }^{10}$ à la Franche-Comté où Jean Boichard signale que les bâtiments traditionnels «situés d'une manière très incommode, au beau milieu du village » font obstacle à toute transformations in situ ${ }^{11}$, en passant par les Ardennes où Jeanne Bérenguer évoque l'obligation d'un remodelage complet des villages ardennais et lorrains pour accueillir la modernisation ${ }^{12}$, toutes les observations convergent vers un constat sans appel : l'incapacité des structures anciennes à répondre aux exigences de l'élevage moderne. Des monographies de René Dumont (1951) aux thèses de géographie des années 1960 à 1980, les auteurs fustigent l'archaïsme des structures héritées qui freinent le progrès en marche.

Le constat unanime de l'inadaptation fonctionnelle du bâti hérité conduit les auteurs à s'interroger sur les formes de changement. Le remplacement de constructions anciennes par des neuves n'est pas la règle: des adaptations transitoires reculent le moment de l'investissement. Dans certains cas, des modifications de détail suffisent pour redonner vie aux anciennes structures. Dans le centre du Bassin parisien, « certaines fermes isolées sont si vastes que les écuries et les étables devenues inutiles peuvent être transformées en locaux d'ensilage et en garage pour le parc de machines $»^{13}$. Dans les pays de petite et moyenne exploitations viticoles comme la Bourgogne ou les Côtes-du-Rhône ou dans les régions de polyculture et d'habitat semi-dispersé en hameaux comme le Sud-Ouest ou le Massif central, les exploitants rachètent les bâtiments attenants de ceux qui ont quitté la terre et les transforment. Cependant, pour les nouvelles spéculations agricoles, l'opération s'avère le plus souvent impossible : installer un silo de stockage des récoltes ou de la nourriture du bétail, une porcherie, une batterie de volailles nécessite un nouveau chantier.

Dans les années 1960, ceux qui se lancent dans des chantiers de construction sont encore peu nombreux à cause de la priorité des investissements en matériel. Pour le plus grand nombre, les dépenses consacrées à l'achat du tracteur ne laissent plus de place à d'autres dépenses d'envergure. Au regard d'investissements mobiliers prioritaires (mécanisation et motorisation), la modernisation agricole se résume à l'adjonction de bâtiments autoconstruits et à l'adaptation au coup par coup des édifices existants. Même pour la production avicole naissante, Jean-Paul Diry procède au même constat: "Dans un premier temps, les nouveaux aviculteurs ont utilisé de vieilles granges, des greniers, voire les étables libérées pendant la belle saison » [mais] " quelques-uns, très vite imités dans les régions où l'aviculture se développe, se résolvent alors à construire des poulaillers de bois, couverts en papier goudronné. [...]. Dès 1958-1959, les poulaillers en brique, en parpaing et surtout en fibrociment se multiplient. Dès le début des années 1960, les ateliers de 10000 têtes sortent de terre ${ }^{14} »$. 

rural, toutes les thèses de géographie de l'entre-deux-guerres avaient fait un sort à la maison paysanne. La démarche perdure dans des travaux des années 1950 et 1960 : les structures anciennes y sont décrites comme les vestiges d'un monde rural en proie à l'exode et à la modernisation accélérée de l'agriculture. Alors pourquoi les géographes, si sensibles aux formes matérielles de l'habitat, ont-ils si peu étudié les nouvelles constructions agricoles? Deux registres d'explication sont proposés : des raisons liées aux sources d'une part, des raisons disciplinaires d'autre part.

\section{Le bâtiment agricole : un objet géographique peu visible dans les statistiques publiques}

17 Après-guerre, les préoccupations des géographes convergent vers les transformations introduites par les aides à la reconstruction agricole et par les politiques de modernisation des structures. On scrute les restructurations parcellaires introduites par le remembrement; on étudie la modernisation des systèmes de production et la concentration des exploitations. On décrit les changements repérés grâce à l'utilisation des intrants chimiques; on analyse la motorisation des activités agricoles et ses effets. Que ce soit dans l'ouvrage de Daniel Faucher intitulé Le paysan et la machine (1954) ou, dix ans plus tard, dans les 20 pages que consacre Roger Brunet à " la révolution du tracteur » dans sa thèse sur les campagnes toulousaines ${ }^{16}$, rien de ce thème n'échappe aux auteurs. Nombre de ces orientations sont devenues possibles grâce à la disponibilité de données quantitatives qui émanent des nouveaux organismes nationaux de statistiques. Elles donnent lieu à des synthèses dans lesquelles les réalisations cartographiques soulignent les disparités spatiales dans la diffusion du tracteur, la consommation d'engrais, l'étendue des superficies irriguées ${ }^{17}$. Dans ce contexte, la maison traditionnelle du paysan devient un enjeu de recherche historique et ethnologique. Le corps de ferme - ou plus exactement l'exploitation agricole - engagé dans la voie du productivisme agricole fait-il l'objet de plus de sollicitude?

Alors que les géographes s'appuient de plus en plus sur la statistique officielle pour étayer leur analyse des mutations, les renseignements disponibles sur les constructions rurales sont décevants. Dans le recensement général agricole de 1955 comme dans celui de 1970,

In Situ, 21 | 2013 
les données relatives aux bâtiments d'exploitation restent discrètes. À partir de sondages (1962), de recensements spécifiques (1968), on dispose toutefois de chiffres sur l'évolution du nombre de poulaillers et plus généralement des ateliers hors sol comme l'indique JeanPaul Diry pour la Bretagne ${ }^{18}$. On recense aussi la présence d'équipements des exploitations : l'électricité - dont la force -, l'adduction d'eau (individuelle et collective), la salle de traite avec sa trayeuse mécanique. Maigres références qui s'adressent principalement aux exploitations d'élevage laitier et qui ne peuvent suffire à l'examen exhaustif des évolutions!

L'enquête "bâtiment", réalisée par le ministère de l'Agriculture pendant l'hiver 1965-1966auprès d'un échantillon de 200 à 250 exploitations par département, n'apporte pas plus de satisfactions. Devant la rapidité des transformations du secteur agricole, les résultats, publiés une décennie plus tard (1975), présentent une valeur historique incontestable que les géographes n'exploitent guère ${ }^{19}$. Les raisons en sont simples : même si des publications partielles ont été réalisées en 1970 et 1971, les dix années qui se sont écoulées entre la réalisation des enquêtes et leur publication ont enregistré d'intenses transformations des exploitations. Surtout, les résultats de l'enquête ont donné lieu à une cartographie aux échelles régionale et départementale qui convenait peu aux pratiques méthodologiques. La synthèse à laquelle elle aboutit, pour l'ensemble des rubriques, n'en n'est pas moins intéressante (document 1).

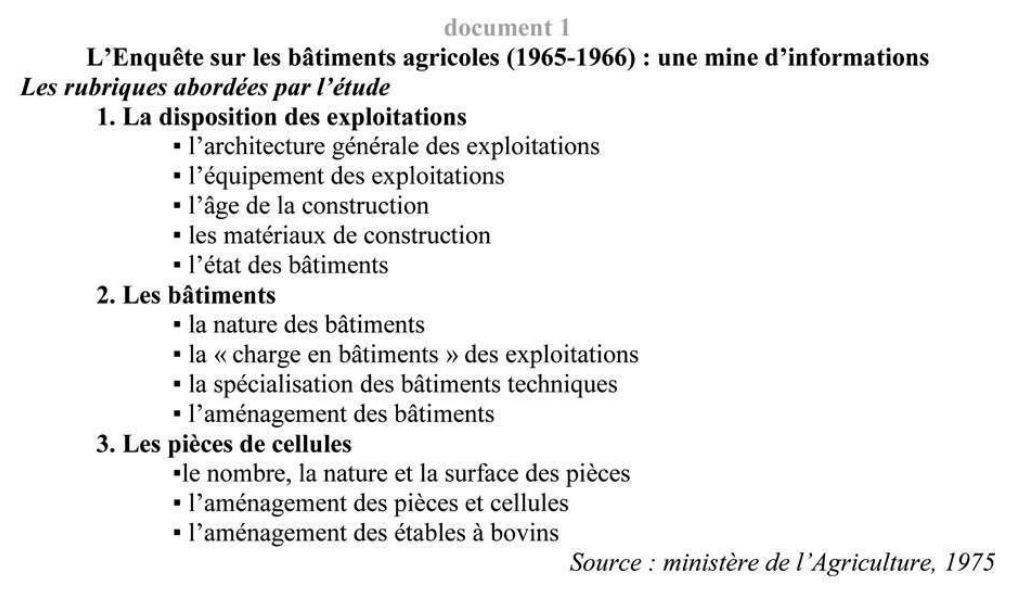
traduisent bien l'obsolescence d'un bâti que les agriculteurs rompus au discours de la Jeunesse agricole catholique et formés dans les centres agricoles d'études techniques (c eta) considèrent comme inadapté aux besoins d'une agriculture moderne. Au contraire, le dynamisme de certains départements s'affiche : c'est le cas de la Bretagne engagée très tôt dans la spécialisation hors sol ${ }^{20}$.

21 Pour les chercheurs, la quantification est donc assez limitée. Ainsi, dans les travaux qui rendent compte de la dynamique spatiale des mutations à l'échelle nationale, les données sur le bâti font défaut. Dans les typogrammes élaborés par l'équipe de géographie rurale de l'Institut de géographie ${ }^{21}$ comme dans les typologies réalisées dans les décennies suivantes, seules figurent des données relatives à l'orientation des productions, à la productivité, à la concentration des exploitations, à la commercialisation, aux aspects techniques et à bien d'autres encore au fur et à mesure de la sophistication des moyens 
mis en œuvre. Il faut attendre la mise en place d'une politique de soutien public à la construction agricole (1966) et plus tard, les fichiers des permis de construire fournis par le ministère de l'Équipement pour analyser, à l'échelle nationale, la dynamique de construction. Pour l'heure, la faible disponibilité statistique et le manque de fiabilité des données ${ }^{22}$ obligent les chercheurs à s'en remettre à des analyses de cas qu'ils réalisent lors de leurs enquêtes de terrain.

Ces monographies d'exploitation constituent de précieux documents pour rendre compte des transformations en cours: les travaux d'Armand Frémont (1967), de Jean-Pierre Houssel (1978), de Jeanne Dufour (1981), de Georges Macé (1985), de Jean-Paul Diry (1985) et enfin de Corentin Canévet (1992) en témoignent. La liste des auteurs cités ne trompe pas : tous ont placé l'élevage comme cible principale de leur enquête ou lui accordent tout au moins un rôle important. Ces exemples procurent des témoignages forts de l'évolution des structures dans laquelle la construction prend toute sa place à travers les dimensions organisationnelle (l'amélioration des conditions de vie), économique (élargissement du troupeau et amélioration de la productivité), sociale (entre ceux qui peuvent investir et ceux qui adaptent l'existant) et sanitaire. Accompagnées le plus souvent de photographies et de croquis, ces monographies d'exploitation apportent des preuves matérielles de la métamorphose des maisons paysannes en « fermes modernes » (fig. $\mathbf{n}^{\circ} \mathbf{1}, \mathbf{n}^{\circ} \mathbf{2}$ et $\left.\mathbf{n}^{\circ} \mathbf{3}\right)$.

Figure 1

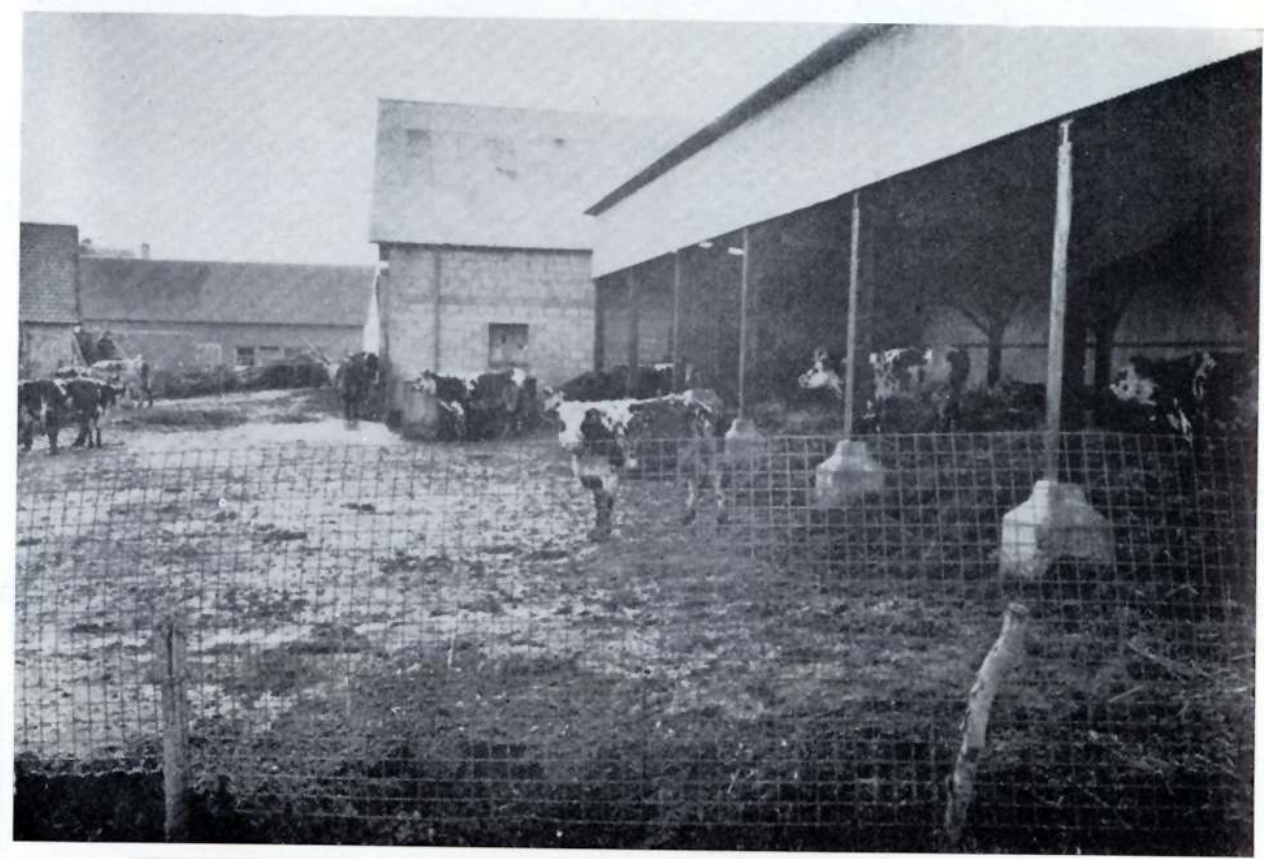

FIGURE 11. - Petite stabulation libre d'un G.A.E.C. du Bocage

(cliché J. Windenberger)

Une petite stabulation libre dans un GAEC du Bocage normand. Source : FRÉMONT, Armand. L'Élevage en Normandie. Étude géographique. Caen : Association des publications de la faculté des Lettres et Sciences humaines de l'université de Caen, 1967, vol. 1. (p. 284 bis).

PHOT. WINDENBERgER J. ( ) J. WINDENBERgER. 
Figure 2

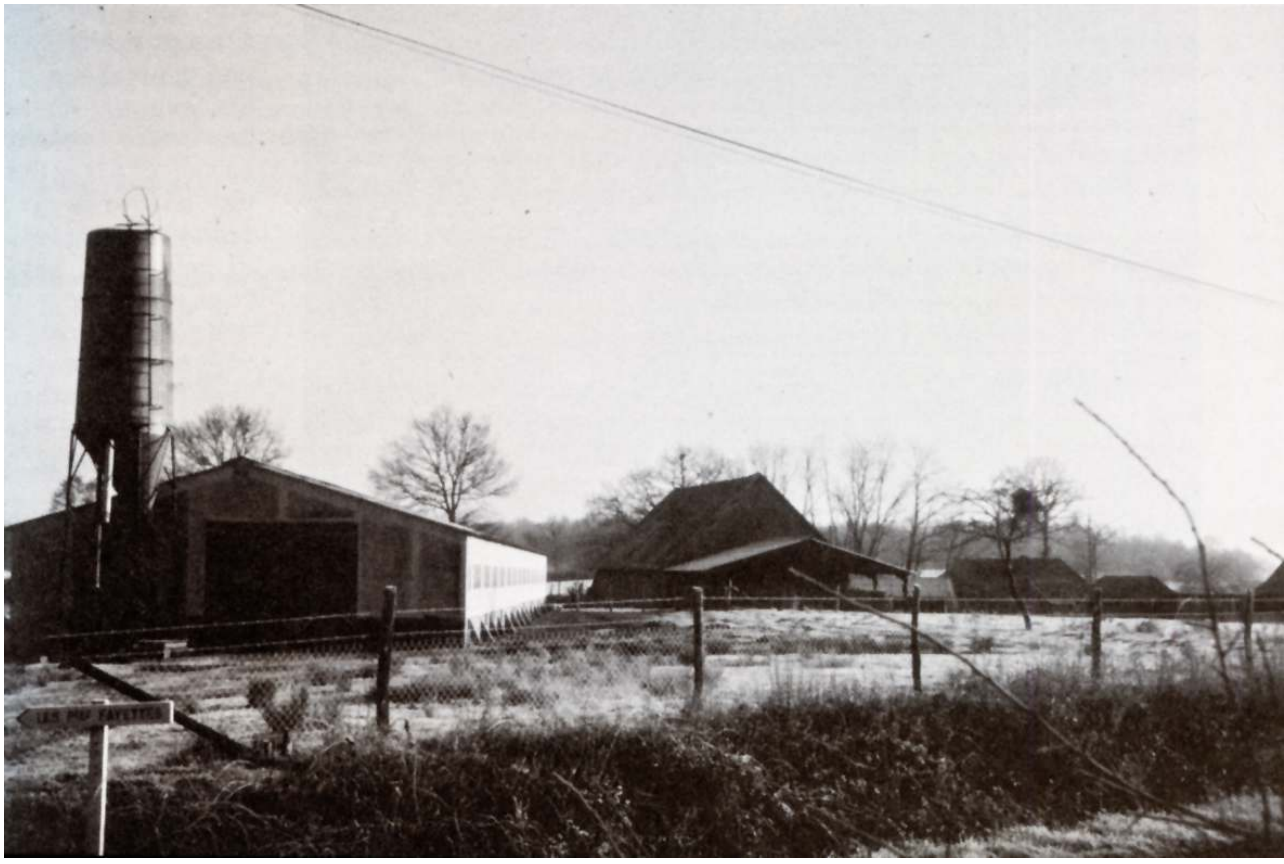

Un poulailler en fibrociment dans l'Allier. Source : DIRY, Jean-Paul. L'Industrialisation de l'élevage en France. Économie et géographie des filières avicoles et porcines. Gap : éditions Ophrys, 1985, p. 101 Phot. Diry, Jean-Paul. (c) Jean-Paul Diry. 
Figure 3
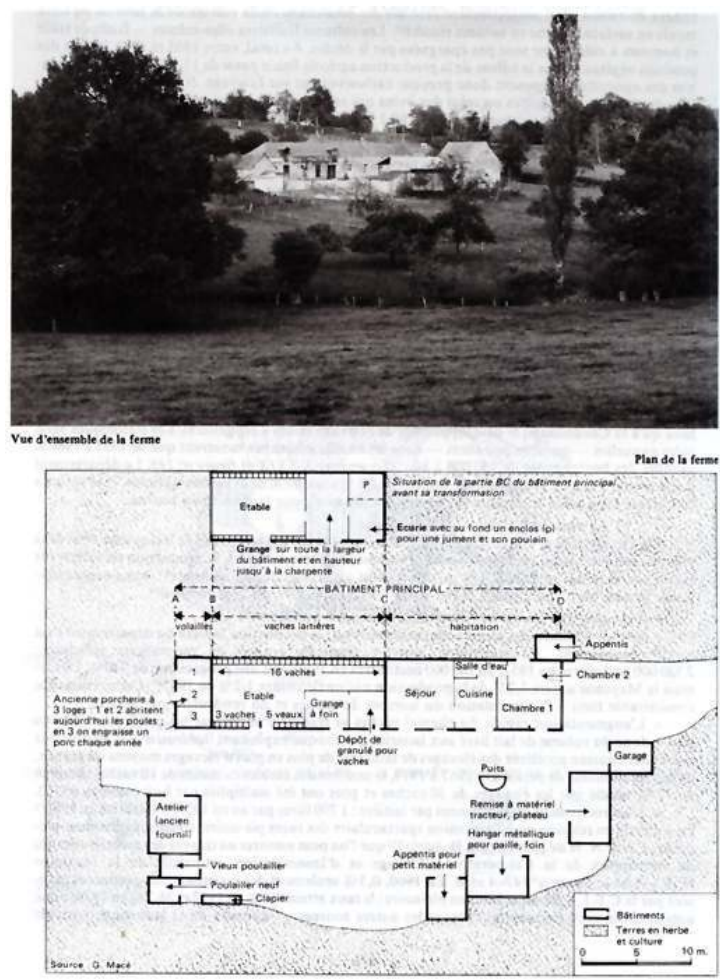

L'exploitation de la Bodinière (18,5 ha) dans le canton de Chailland en 1977. Vue d'ensemble de la ferme et plan de la ferme. Source : MACÉ, Georges. Un département rural de l'Ouest. La Mayenne. Mayenne : Joseph Floch Éd., 1982, p. 432.

PHOT. MACÉ, GEORgES. (C) GEORgES MACÉ.

À la lecture des thèses de géographie rurale qui consacrent des chapitres à la modernisation de l'agriculture, la part accordée à la construction agricole est ténue. L'absence de statistiques suffisamment étoffées en rend compte pour une part. Mais dans cette approche des vecteurs de la modernisation, on aurait tort d'expliquer la moindre place accordée à la construction agricole par rapport à celle du tracteur avec les seuls arguments statistiques. Des changements propres à la discipline peuvent aussi expliquer cette relative désaffection.

\section{Construction agricole et géographie : les raisons d'un désintérêt}

Dans le contexte général d'une "mathématisation» des recherches, les statistiques contribuent à mettre en avant ou, au contraire, à réduire la visibilité des objets étudiés. Ainsi, contrairement aux données sur la motorisation des exploitations, la construction agricole se fait discrète dans les chapitres des thèses relatives à la modernisation des fermes. On le conçoit bien pour les exploitations spécialisées dans les productions végétales mais pas pour les structures d'élevage. Cette absence est, comme on l'a déjà avancé, avant tout liée à la disponibilité des sources. Le constat vaut jusqu'au milieu des années 1960. À partir de cette date, les résultats de "l'enquête bâtiments » diligentée par le ministère de l'Agriculture sur l'ensemble des départements conduit les pouvoirs publics à encourager la construction neuve. Désormais ces derniers inscrivent la construction agricole parmi les clés de la modernisation des exploitations. Du décret du 
25 mai 1966 jusqu'à l'actuel Plan de modernisation des bâtiments d'élevage, les constructions agricoles bénéficient du soutien continu des pouvoirs publics. Le calendrier des premières aides en témoigne :

25 - décret du 25 mai 1966 : subvention des bâtiments de 15 UGB $^{23}$ minimum à hauteur de $40 \%$ pour une construction atteignant au maximum 30000 francs ;

26 - 1971 : le barème des subventions à la construction neuve change pour un forfait par animal logé et par type de production: 500 francs par vache laitière ou à viande, 150 francs par brebis laitière, 120 francs par brebis à viande. Des montants supérieurs sont alloués aux constructions de montagne (ex. : 1500 francs pour une vache laitière) ;

27 - 1974 : suppression des subventions en dehors des zones de rénovation rurale. Possibilité d'emprunt dans le cadre de « prêts spéciaux d'élevage »;

28 - 1977 : le forfait de la subvention par animal logé est abandonné. Désormais, un plafond de dépense est fixéet une différence introduite entre les exploitations inscrites dans un plan de développement de celles qui ne lesont pas ;

29 -après 1978, les zones de plaine sont exclues des subventions ${ }^{24}$. Ailleurs, elles sont plafonnées à $20 \%$ des dépenses. La baisse du plafond de subventionnement est compensée, dans les zones rurales fragiles et les zones de montagne, par une subvention du Fidar (Fonds interministériel de développement et d'aménagement rural) ;

30 - dans le cadre de la décentralisation, le soutien à la construction agricole apparaît clairement comme une priorité pour certaines régions. En Midi-Pyrénées, la convention État-Région (1982-1983) comporte un plan de relance des bâtiments d'élevage qui en fait une priorité pour le développement agricole régional. 
Figure 4

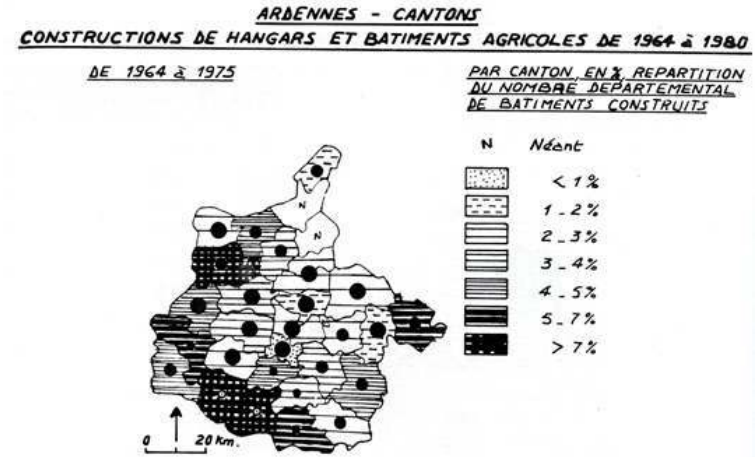

NOMBRE MOYEN DE BATIMENTS CONSTRUITS \} EN 1970, DE 196* \$ 1975

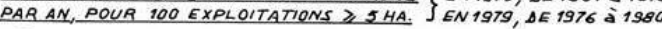

- $<0,50$

$0,50-0,75$

$1-1,50$

$1,50-2$

(3) $2 \quad-2,50$

(4) $2,50-3$

(5) $>3$

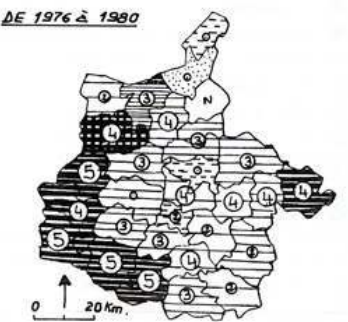

Ardennes : constructions de hangars et de bâtiments agricoles par cantons entre 1964 et 1980.

Source : BÉRENGUER, Jeanne. De l'Ardenne à l'Argonne. Progrès et régressions dans le monde agricole, 1985, p. 291

Phot. Bérenguer, Jeanne. (C) Jeanne Bérenguer.

31 La confection d'un dossier d'instruction pour l'octroi de subventions à la construction génère des statistiques publiques que les géographes s'empressent de solliciter. Celles-ci donnent lieu à des productions cartographiques à différentes échelles. Dans ses travaux sur les Préalpes de Haute-Provence, André de Réparaz publie une carte de localisation des bergeries subventionnées entre 1966 et 1976 (fig. nº). Dans les Ardennes, Jeanne Bérenguer propose en 1985 une cartographie en valeurs relatives, des hangars et des bâtiments subventionnés à l'échelle cantonale (fig. $\mathbf{n}^{\circ} 5$ ). Le constat semble évident. Dès qu'ils ont en main des statistiques publiques leur permettant la confection de cartes, les géographes s'emparent de données qui confortent leur analyse des dynamiques en cours. Une carte de synthèse nationale réalisée par Aliette Delamarre pour la période 1966-1970 en atteste (fig. $\mathbf{n}^{\circ} \mathbf{6}$ ). Dans l'élevage bovin comme dans celui des porcs, la dynamique de construction identifie les besoins des structures pour l'ensemble des régions et révèle, par l'ampleur des subventions, les zones de spécialisation telles que la Bretagne pour la production porcine. 
Figure 5

Bergeries nouvelles "subventionnees" $1966-76$

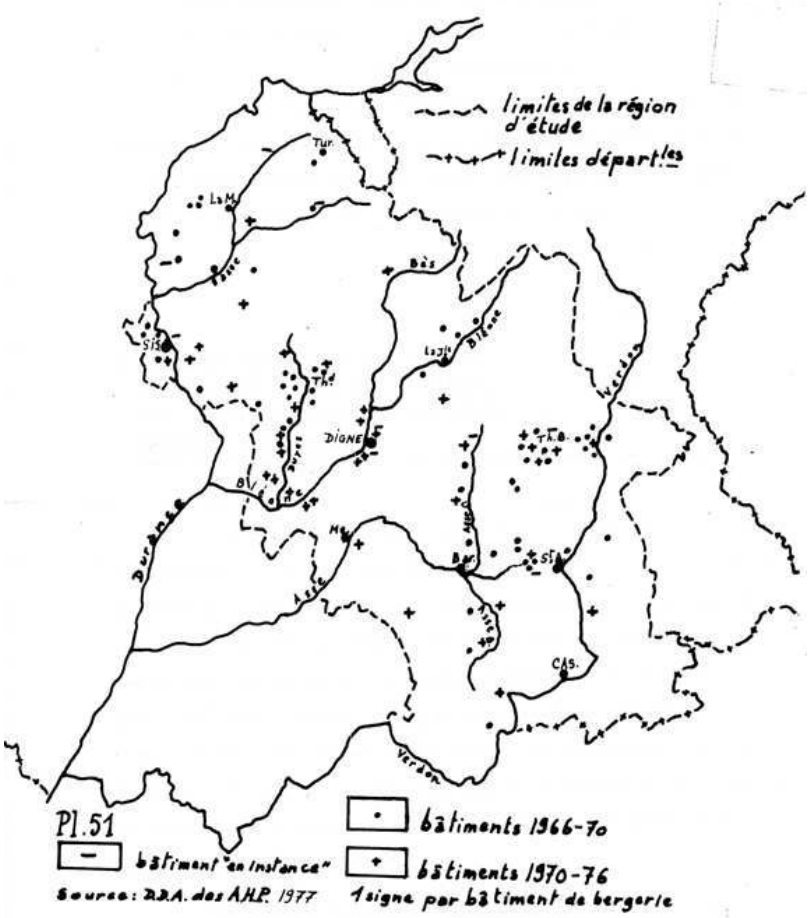

Les bergeries subventionnées dans les Préalpes de Haute-Provence entre 1966 et 1976. Source: RÉPARAZ, André de. La Vie rurale dans les Préalpes de Haute-Provence, 1978, p. 1201.

Phot. Réparaz, André de. () André de Réparaz. 


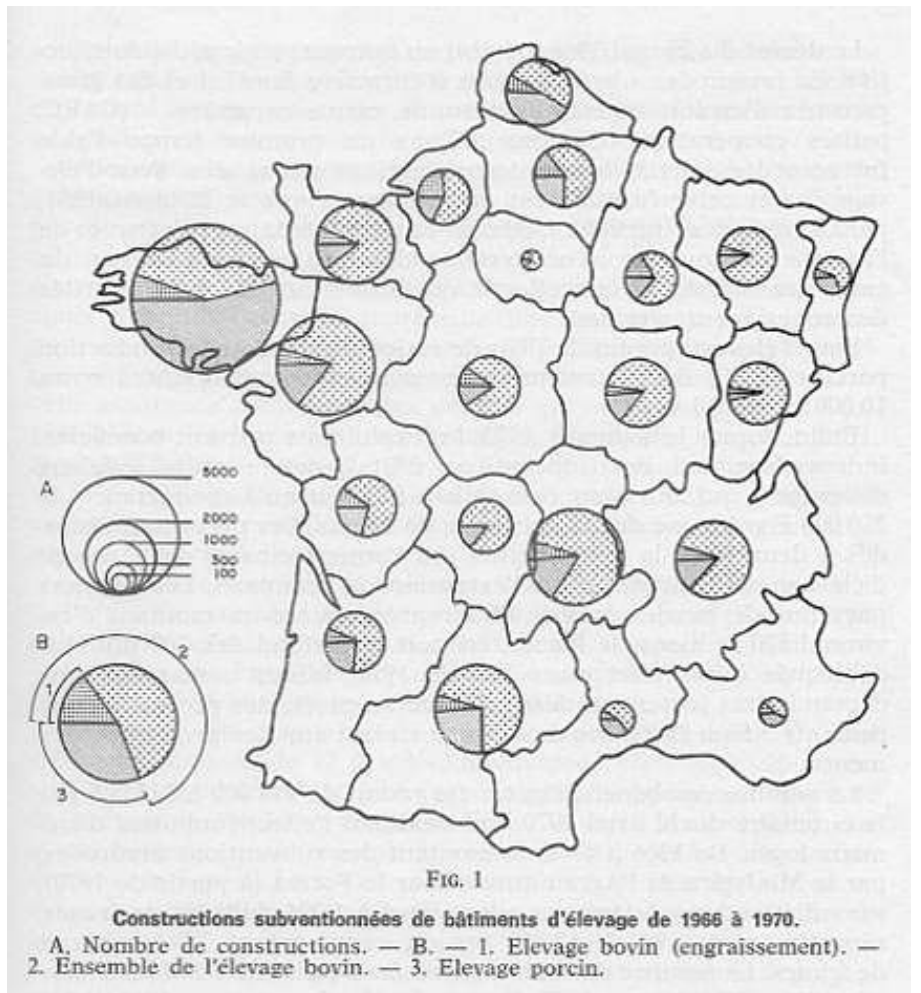

Bâtiments d'élevage subventionnés de 1966 à 1970. Source : DELAMARRE, Aliette. « Les bâtiments modernes d'élevage en France ». Revue géographique des Pyrénées et du Sud-Ouest, t. 47, fasc. 2, Toulouse, 1976, p. 142.

Phot. Delamarre, Aliette. (c) Aliette Delamarre.

Ce premier point apporte des arguments pour comprendre les raisons du faible traitement de la construction agricole par rapport à des thèmes beaucoup mieux renseignés par la statistique. Mais un autre aspect mérite toute notre attention: la rupture épistémologique et générationnelle dont les effets mettent en avant ou minimisent, au contraire, certains objets géographiques.

\section{Des ruptures épistémologiques et générationnelles}

Il est difficile de comprendre la faiblesse des analyses du bâti contemporain dans la modernisation agricole sans faire référence aux changements qui s'opèrent dans la discipline au tournant des années 1960-1970. En effet, pour toute une génération de géographes baignée dans la géographie classique pour laquelle la maison paysanne était considérée comme un élément identitaire des campagnes, la sensibilité aux nouvelles constructions érigées dans les campagnes est faible. Ces constructions aux formes et aux matériaux standardisés et industrialisés créent une rupture paysagère forte. Cette standardisation des matériaux de construction frappe les chercheurs : celle-ci touche les formes, les coloris des matériaux et le volume. Pour Roger Calmès qui analyse les campagnes des Ségalas et du Lévezou, c'est un moyen de différencier les phases principales de la modernisation: "L'économie traditionnelle a engendré des bâtiments d'exploitation aux toitures de schistes (les lauzes). [...] Une partie de ces bâtiments a disparu pour être remplacée par les toits rouges dans la période d'après-guerre. [...] Dès le 
début des années 1960 apparaissent les toits blancs (éverite). Leur apparition est liée à l'industrialisation de la construction et à l'investissement énorme consenti par les agriculteurs éleveurs. Le progrès récent peut s'identifier aux toits blancs ${ }^{25}$. »

Pour d'autres, plutôt minoritaires, le progrès incarné par la stabulation libre renvoie à une dimension esthétique: "Les étables vétustes abritent, pour quelque temps encore, quelques groupes d'animaux en attendant que la nouvelle stabulation soit entièrement aménagée. Cette élégante bâtisse, avec ses grosses poutres cintrées en sapin verni, ses pignons recouverts de matériaux collés, ses auvents débordants, dont les chevrons sont dissimulés sous une "frisette » cirée, ressemble un peu à un chalet comtois et, par son existence-même, atteste qu'on peut tout à la fois construire du fonctionnel et du beau ${ }^{26}$. »

À de rares exceptions près, pour de nombreux géographes qui avaient scruté l'ordonnancement de "beaux paysages $»^{27}$, le bouleversement du bâti traditionnel soulève la difficile cohabitation paysagère entre constructions traditionnelles et nouvelles. Pour certains, l'intensité des changements conduit même à évacuer l'intérêt géographique de l'objet. C'est ce qui ressort des propos de Max Derruau: «Les maisons rurales tendent de plus en plus à s'uniformiser, du moins dans le cadre de chaque État moderne. [...] La facilité des transports répand un même type en de nombreux exemplaires qui ne diffèrent que par des traits contingents. La ferme américaine à cour ouverte, séparant le logement, l'immense grange et d'autres bâtiments, parmi lesquels un hangar, n'a pas plus de signification géographique qu'une villa de nos banlieues ${ }^{28}$.»

Lors des années d'après-guerre, la visibilité des constructions agricoles dans la recherche géographique n'a sans doute pas été à la hauteur de leur importance dans les transformations sociales et économiques des structures d'exploitation, notamment pour les activités d'élevage. Deux éléments d'explication peuvent être retenus. Pour toute une génération de chercheurs rompue à une géographie de la maison paysanne traditionnelle, la mise en chantier de bâtiments standardisés détruit l'ancien ordonnancement du bâti jusqu'à en réduire l'intérêt géographique. Pour la nouvelle génération engagée dans une géographie théorique et quantitative, la scientificité d'un objet d'étude repose en partie sur l'analyse mathématique: l'absence de statistiques suffisamment étoffées sur les constructions agricoles neuves a conduit bon nombre de géographes à se tourner vers d'autres sources d'analyse de la modernisation.

\section{Conclusion}

Dans la modernisation agricole de la seconde moitié du $\mathrm{XX}^{\mathrm{e}}$ siècle, les géographes ont montré, dans la continuité des travaux sur la maison paysanne, toute l'importance des bâtiments d'exploitation. Dans le même temps, les pouvoirs publics n'ont pas ignoré les pesanteurs du bâti agricole traditionnel dans la dynamique engagée : la démonstration en est faite à travers une grande enquête menée au début des années 1960. Partout en France, et surtout dans les zones spécialisées dans l'élevage, le travail de terrain des géographes a confirmé le constat. Toutefois, dans les thèses d'État soutenues du début des années 1950 au début des années 1990, la place accordée à la construction agricole comme facteur de modernisation des structures de production n'a sans doute pas bénéficié de toute l'attention nécessaire. Dans leurs enquêtes, les chercheurs montrent bien que le bâti de l'exploitation traditionnelle, pourtant modifié au gré de l'évolution des structures, se révèle un obstacle pour une agriculture performante. La ferme moderne qui permet d'y parvenir est une structure adaptée à de nouvelles conditions de production. En 
privilégiant l'agrandissement des structures, la diffusion du matériel motorisé et la spécialisation des productions, les pouvoirs publics encouragent un renouveau des constructions agricoles qui s'effectue, le plus souvent, par étapes. Les chercheurs ne parviennent que difficilement à l'analyser. En effet, contrairement à la diffusion du tracteur et des engrais que les géographes ont pu suivre à la trace grâce à la profusion des statistiques, le bâti agricole n'a pas bénéficié du même traitement. Cette relégation s'est perpétuée jusqu'au moment où des politiques de soutien à la construction ont produit des dossiers permettant de cartographier, aux échelles départementale, cantonale et communale, la dynamique de construction. Alors que la discipline laissait derrière elle l'approche vidalienne pour privilégier l'analyse des statistiques publiques, les chercheurs ont privilégié certains vecteurs de la modernisation. À ce moment de l'évolution géographique où les préoccupations paysagères ne focalisent pas encore l'attention, les analyses du nouveau bâti soulèvent des questions: quelle priorité en termes d'investissements entre la stabulation libre et l'accès au confort domestique? Quel devenir des constructions anciennes rendues obsolètes? Au total, il est indéniable que les constructions agricoles contemporaines ont joué un rôle beaucoup plus important que ne le laisse penser la littérature existante.

\section{BIBLIOGRAPHIE}

\section{Le corpus des thèses}

BÉRENGUER, Jeanne. De l'Ardenne à l'Argonne. Progrès et régressions dans le monde agricole. Thèse de doctorat d'État en géographie, université Lyon III, 1985, 847 p. (dactylographiée).

BÉTEILLE, Roger. Les Aveyronnais. Essai géographique sur l'espace humain. Poitiers, 1974. Imprimerie de l'Union, $573 \mathrm{p}$.

BOICHARD, Jean. «L'élevage bovin en Franche-Comté ». Université Paris IV. Cahiers de géographie de Besançon, $\mathrm{n}^{\circ} 26$ Paris : Les Belles lettres, 1977, $536 \mathrm{p}$.

BONNAMOUR, Jacqueline. Le Morvan. La terre et les hommes. Essai de géographie agricole. Paris :PUF, $1965,454 \mathrm{p}$.

BOUET, Guy. L'Évolution récente de la vie rurale en Limousin. Lille, Atelier de reproduction des thèses, $1979,669 \mathrm{p}$.

BOZON, Pierre. La Vie rurale en Vivarais. Étude géographique, Valence-sur-Rhône, 1963, 641 p.

BRUNET, Pierre. Structure agraire et économie rurale des plateaux tertiaires entre la Seine et l'Oise. Caen : Société d'imprimerie Caron, 1960, 552 p + 7 pl. hors texte.

BRUNET, Roger. Les Campagnes toulousaines. Étude géographique. Toulouse : Publication de la faculté des Lettres et Sciences humaines de Toulouse, 1965, Série B, t. 1.

CALMÈS, Roger. Les Campagnes des Ségalas et du Lévezou. Développement et progrès agricoles. Rodez : éditions Subervie, 1980, $226 \mathrm{p}$. 
CANÉVET, Corentin. Le Modèle agricole breton : histoire et géographie d'une révolution agro-alimentaire. Rennes : PUR, 1992, 397 p.

CHAPUIS, Robert. Les Ruraux du Doubs. Éléments de géographie sociologique. Besançon : Cêtre, 1982, $388 \mathrm{p}$.

CHEVALIER, Jacques. La Question foncière en Basse-Normandie. Essai de géographie des rapports sociaux à propos du foncier agricole. Thèse de doctorat d'État, université de Caen, 1983, 677 p.

(dactylographiée).

DAVEAU, Suzanne. Les Régions frontalières de la montagne jurassienne. Étude de géographie humaine. Lyon : publication hors série de la revue de géographie de Lyon, Mémoires et Documents, $\mathrm{n}^{\circ} 14$, $1959,571 \mathrm{p}$.

DIRY, Jean-Paul. L'Industrialisation de l'élevage en France. Économie et géographie des filières avicoles et porcines. Gap : éditions Ophrys, 1985, $680 \mathrm{p}$.

DUFOUR, Jeanne. Agriculture et agriculteurs dans les campagnes mancelles. Le devenir des régions agricoles. Le Mans : chez l'auteur, 1981, 596 p.

DURAND, Alfred. La Vie rurale dans les massifs volcaniques des Dores, du Cézallier, du Cantal et de l'Aubrac. Aurillac : Imprimerie moderne, 1946, 530 p.

FEL, André. Les Hautes Terres du Massif central. Tradition paysanne et économie agricole. ClermontFerrand : publications de la faculté des Lettres et Sciences humaines de Clermont-Ferrand, Institut de géographie, $\mathrm{n}^{\circ} 23,1962,340 \mathrm{p}$.

FRÉMONT, Armand. L'Élevage en Normandie. Étude géographique. Caen : Association des publications de la faculté des Lettres et Sciences humaines de l'université de Caen, 1967, 2 vol., 626 p. et 316 p.

HOUSSEL, Jean-Pierre. Le Roannais et le Haut-Beaujolais. Un espace à l'écart des métropoles. Lyon : Presses universitaires de Lyon, 1978, 238 p.

JUILLARD, Étienne. La Vie rurale dans la plaine de Basse-Alsace. Essai de géographie sociale. Strasbourg : éditions F. X. Le Roux, 1953, 582 p.

LEBEAU, René. La Vie rurale dans les montagnes du Jura méridional. Étude de géographie humaine. Institut des études rhodaniennes de l'université de Lyon, publication hors série de la revue de géographie de Lyon, Mémoires et Documents, $n^{\circ}$ 9, 1955, 693 p.

LERAT, Serge. Les Pays de l'Adour. Structures agraires et économie agricole. 1963, 578 p.

LIVET, Roger. Habitat rural et structures agraires en Basse-Provence. Publications des annales de la faculté des Lettres d'Aix-en-Provence, 1962, 465 p.

MACÉ, Georges. Un département rural de l'Ouest. La Mayenne. Mayenne : Joseph Floch Éd., 1982, 2 t., $1011 \mathrm{p}$.

MOREAU, Jean-Pierre. La Vie rurale dans le Sud-Est du Bassin parisien entre les vallées de l'Armançon et de la Loire. Étude de géographie humaine, publications de l'université de Dijon, XVII. Paris : Société Les Belles Lettres, 1959, 339 p.

RENARD, Jean. Les Évolutions contemporaines de la vie rurale dans la région nantaise. Les Sablesd'Olonne : Le Cercle d'Or, 1976, 432 p.

RÉPARAZ, André de. La Vie rurale dans les Préalpes de Haute-Provence. Thèse d'État en géographie, université Aix-Marseille, 3 t.. Aix-en-Provence : Édisud. Atelier national de reproduction des thèses, université Lille III, $1227 \mathrm{p}$. 
REY, Violette. L'Agrandissement spatial des exploitations agricoles. Thèse d'État en géographie, université Paris I, 1980, 515 p.

ROUDIÉ, Philippe. Campagnes girondines et vins de Bordeaux à l'époque contemporaine. Bordeaux : éditions du CNRS, 1980, $436 \mathrm{p}$.

SOLLE, Henriette. Un pays rural dans l'orbite de Paris : Le Gâtinais. Thèse de doctorat d'État en géographie, université Paris IV, 1976, 698 p. (dactylographiée).

\section{Autres titres}

BERGER, Martine, GILETTE, Chantal, et ROBIC, Marie-Claire. «L'étude des espaces ruraux en France à travers trois quarts de siècle de recherche géographique. L'exemple des thèses de doctorat d'État ». Strates, n 9, 1996-1997, p. 133-164 (première parution en 1975 dans Réflexions sur l'espace rural français, approches, définitions, aménagement, université Paris I-ENS Fontenay-auxRoses, septembre 1975, p. 3-51).

BONNAMOUR, Jacqueline, (dir.). Étude géographique des exploitations agricoles. Réflexion et propositions méthodologiques. Laboratoire associé de Géographie humaine de l'Institut de géographie de Paris, équipe de géographie rurale, rapport n 1, avril 1972, 162 p. Géographie rurale. Méthodes et perspectives. Paris : Masson, 1973, 168 p.

DELAMARRE, Aliette. « Les bâtiments modernes d'élevage en France ». Revue géographique des Pyrénées et du Sud-Ouest, t. 47, fasc. 2, Toulouse, 1976, p. 139-158.

DEFFONTAINES, Jean-Pierre, et OSTY, Paul-Louis. « Des systèmes de productions agricole aux systèmes agraires ». L'Espace Géographique, n 3, 1977, p. 195-199.

DERRUAU, Max. Nouveau Précis de géographie humaine. Paris : A. Colin, 1971, 575 p.

DIRY, Jean-Paul. Les Espaces ruraux. Paris : Sedes, 1999, 192 p.

LIVET, Roger. Les Nouveaux Visages de l'agriculture française. Paris : éditions Économie et Humanisme, 1980, 210 p.

MADELINE, Philippe. « L'évolution du bâti agricole en France métropolitaine : un indice des mutations agricoles et rurales ». L'Information géographique, septembre 2006a, $\mathrm{n}^{\circ}$ 3, vol. 70, p. 33-49. « Les constructions agricoles dans les campagnes françaises. Héritages et dynamiques actuelles d'évolution ». Histoire et Sociétés rurales, $\mathrm{n}^{\circ} 26,2^{\mathrm{e}}$ semestre, 2006b, p. 53-93. Construction agricoles d'hier et d'aujourd'hui. Essai de géographie sociale. Habilitation à diriger des recherches, Nantes, 2007, 336 p. MADELINE, Philippe et MORICEAU, Jean-Marc. Bâtir dans l'espace rural, Les enjeux de la construction de la protohistoire au XXI siècle. Bibliothèque du Pôle rural, hors série des Enquêtes Rurales, 1, Pôle rural de la MRSH, Caen, 2007, 368 p.

Ministère de l'Agriculture. Enquête sur les bâtiments d'habitation et d'exploitation agricoles (1965-1966). Étude ${ }^{\text {os }} 135$ et 135 bis, coll. «Statistiques agricoles », 1975, t. 1, 172 p. et t. 2, annexe, 209 p.

PITTE, Jean-Robert. Histoire du paysage français. De la préhistoire à nos jours. Paris : Tallandier, $3^{\mathrm{e}}$ éd., 2003, $444 \mathrm{p}$.

\section{NOTES}

1. - DIRY, Jean-Paul. Les Espaces ruraux. Paris : Sedes, 1999, p. 187.

2. - CALMÈS, Roger. Les Campagnes des Ségalas et du Lévezou. Développement et progrès agricoles. Rodez : éditions Subervie, 1980, p. 138. 
3. - FRÉMONT, Armand. L'Élevage en Normandie. Étude géographique. Caen: Association des publications de la faculté des Lettres et Sciences humaines de l'université de Caen, 1967, t. 2, p. 217.

4. - DEFFONTAINES, Jean-Pierre, et OSTY, Paul-Louis. « Des systèmes de production agricole aux systèmes agraires ». L'Espace géographique, n 3, 1977, p. 195-199.

5. - Ibid., 1985, p. 37.

6. - Ministère de l'Agriculture. Enquête sur les bâtiments d'habitation et d'exploitation agricoles (1965-1966). Études n ${ }^{\circ} 135$ et 135 bis, coll. «Statistiques agricoles », 1975, t. 1, 172 p. et t. 2, annexe, $209 \mathrm{p}$.

7. - BONNAMOUR, Jacqueline. Le Morvan. La terre et les hommes. Essai de géographie agricole. Paris : PUF, 1965, p. 157 et 159.

8. - DUFOUR, Jeanne. Agriculture et agriculteurs dans les campagnes mancelles. Le devenir des régions agricoles. Le Mans : chez l'auteur, 1981, p. 158.

9. - MACÉ, Georges. Un département rural de l'Ouest. La Mayenne. Mayenne : Joseph Floch Éd., 1982, t. 1, p. 451.

10. - FRÉMONT, Armand. L'Élevage en Normandie. Étude géographique. Caen: Association des publications de la faculté des Lettres et Sciences humaines de l'université de Caen, 1967, vol. 1, p. 185.

11. - BOICHARD, Jean. L'Élevage bovin en Franche-Comté. Université de Paris IV. Cahiers de géographie de Besançon, $\mathrm{n}^{\circ}$ 26. Paris : Les Belles lettres, 1977, p. 472.

12. - BÉRENGUER, Jeanne. De l'Ardenne à l'Argonne. Progrès et régressions dans le monde agricole. Thèse de doctorat d'État en géographie, université Lyon III, 1985, p. 250 à 291.

13. - PITTE, Jean-Robert. Histoire du paysage français. De la préhistoire à nos jours. Paris : Tallandier, 3 e éd., 2003, p. 347.

14. - DIRY, Jean-Paul. L'Industrialisation de l'élevage en France. Économie et géographie des filières avicoles et porcines. Gap : éditions Ophrys, 1985, p. 99.

15. - Ibid., p. 458.

16. - BRUNET, Roger. Les Campagnes toulousaines. Étude géographique. Toulouse : publication de la faculté des Lettres et Sciences humaines de Toulouse, 1965, série B, t. 1, chapitre « La révolution du tracteur », p. 433-454.

17. - LIVET, Roger. Les Nouveaux Visages de l'agriculture française. Paris : éditions Économie et Humanisme, 1980, $210 \mathrm{p}$.

18. - DIRY, Jean-Paul. L'Industrialisation de l'élevage en France. Économie et géographie des filières avicoles et porcines. Gap : éditions Ophrys, 1985, p. 458.

19. - Seuls quelques exemplaires de cette enquête ont été sauvés de la destruction: ils se trouvent aux Archives nationales.

20. - CANÉVET, Corentin. Le Modèle agricole breton: histoire et géographie d'une révolution agroalimentaire. Rennes : PUR, 1992, $397 \mathrm{p}$.

21. - BONNAMOUR, Jacqueline, (dir.). Étude géographique des exploitations agricoles. Réflexion et propositions méthodologiques. Laboratoire associé de Géographie humaine de l'Institut de géographie de Paris, équipe de géographie rurale, Rapport $\mathrm{n}^{\circ}$ 1, avril 1972, $162 \mathrm{p}$.

22. - «Combien de "hangars » bâtis à la hâte, sans permis de construire étaient en fait des poulaillers et des porcheries?», constate Jean-Paul Diry. DIRY, Jean-Paul. L'Industrialisation de l'élevage en France. Économie et géographie des filières avicoles et porcines. Gap : éditions Ophrys, 1985, p. 458.

23. - Unité gros bétail : unité employée pour pouvoir comparer ou agréger des effectifs animaux d'espèces ou de catégories différentes. 1 UGB $=1$ vache de $600 \mathrm{~kg}$ produisant $3000 \mathrm{l}$ de lait par an ; 1 veau de boucherie $=0,5$ UGB ; 1 brebis $=0,15$ UGB (Agreste, 2007).

24. - À cette date, entrée en vigueur du règlement sanitaire départemental qui s'applique aux élevages non concernés par la législation des installations classées. 
25. - CALMÈS, Roger. Les Campagnes des Ségalas et du Lévezou. Développement et progrès agricoles. Rodez : éditions Subervie, 1980, p. 78.

26. - BOICHARD, Jean. "L'élevage bovin en Franche-Comté ", Université de Paris IV, Cahiers de géographie de Besançon, n²6. Paris : Éd. Les Belles lettres, 1977, p. 472.

27. - L'expression est d'E. Sereni, citée par BERGER, Martine, GILETTE, Chantal, et ROBIC, MarieClaire. «L'étude des espaces ruraux en France à travers trois quarts de siècle de recherche géographique. L'exemple des thèses de doctorat d'État ». Strates, n 9, 1996-1997, p. 11.

28. - DERRUAU, Max. Nouveau précis de géographie humaine. Paris : A. Colin, 1971, p. 324.

\section{RÉSUMÉS}

La modernisation agricole de la seconde moitié $\mathrm{du} \mathrm{XX}^{\mathrm{e}}$ siècle a radicalement modifié l'environnement matériel des exploitations. Que l'on aborde la spécialisation des productions, la mécanisation ou la motorisation des activités, les avancées en matière de productivité ou encore l'amélioration des conditions de travail, les géographes ont partout rencontré ces mutations. Parmi celles-ci, ils ont privilégié certains aspects comme les transformations des structures parcellaires ou l'équipement en tracteurs quand d'autres manifestations de la mise en place d'un «modèle» agricole productiviste sont restées beaucoup plus discrètes. Les constructions agricoles en constituent un bon exemple.

Agricultural modernization in the second half of the $20^{\text {th }}$ Century radically changed the hardware environment of farms. Whether one approaches the specialization of production, mechanization or automation activities, advances in productivity or improved working conditions, geographers have met all these changes. Among them, they preferred aspects such as structural transformations parcel or equipment tractors when other manifestations of the establishment of a "model"productivist agriculture remained much more discreet. The farm buildings are a good example.

\section{INDEX}

Mots-clés : modernisation agricole, constructions agricoles, géographie, thèses de doctorat, statistiques

Keywords : agricultural modernization, agricultural buildings, geography, doctoral thesis, statistics

\section{AUTEUR}

\section{PHILIPPE MADELINE}

Professeur de géographie, UFR de géographie - université de Caen-Basse-Normandie, Laboratoire ESO-Caen UMR Espaces et Sociétés 6590 CNRS, Pôle rural de la MRSH de Caen philippe.madeline@unicaen.fr 